\title{
An oncogenic change of function
}

The gene encoding the tricarboxylic acid cycle enzyme isocitrate dehydrogenase $1(\underline{I D H 1})$ is frequently mutated in glioma - the most common type of human brain tumour - but how its mutation contributes to tumorigenesis remains unclear. Shinsan Su and colleagues now present evidence that gliomaassociated $I D H 1$ mutations endow the enzyme with a new activity, causing it to produce the onco-metabolite $R(-)$-2-hydroxyglutarate (2HG).

IDH1 mutations in gliomas are usually missense mutations of the active site arginine 132 (R132), most commonly to histidine. Although it has been shown that R132 substitution impairs the ability

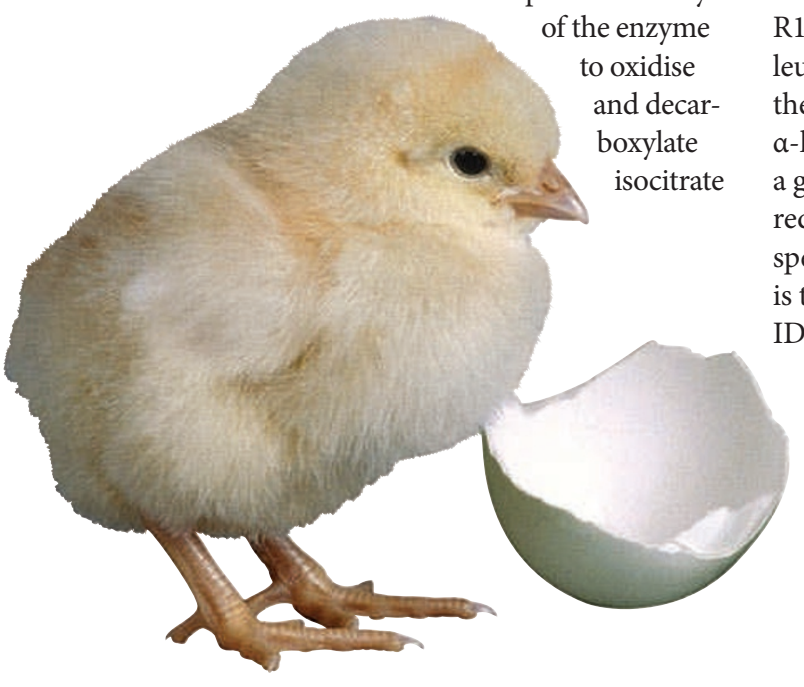

to a-ketoglutarate, a full analysis of the effects on cellular metabolism has been lacking. Using mass spectrometry, Dang et al. carried out an unbiased profile of the metabolites of glioma cell lines stably transfected with the $I D H 1^{R 132 H}$ mutant. Remarkably, the only significant change in metabolites that occurred when $I D H 1^{R 132 H}$ was expressed was an increase of $2 \mathrm{HG}$. Moreover, by examining metabolites from human malignant glioma samples, the authors found that $2 \mathrm{HG}$ production is characteristic of tumours harbouring a range of IDH1-R132 missense mutations.

By examining the enzymatic activity of IDH1-R132 mutants in vitro, the authors discovered that R132 mutation to histidine, cysteine, leucine or serine not only inhibits the conversion of isocitrate to a-ketoglutarate, but also confers a gain-of-enzyme function: the reduction of $a$-ketoglutarate. Mass spectrometry confirmed that $2 \mathrm{HG}$ is the direct product of these mutant IDH1 proteins.

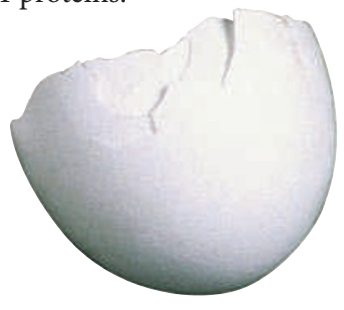

So how is a missense mutation able to change the enzymatic activity of IDH1? To answer this question, the authors solved the crystal structure of the IDH1-R132H mutant. As expected, the structure revealed that the change to histidine resulted in loss of key salt-bridge interactions between the active site of IDH1 and the carboxylates of isocitrate. However, the authors did not anticipate the level of active site reorganization. The positions of both tyrosine 139 and lysine 212, residues thought to be crucial for wild-type IDH1 activity, were shifted in the mutant structure, creating a new active site.

These findings raise the possibility that $2 \mathrm{HG}$ could be a useful prognostic marker of IDH1 mutant gliomas. Although the oncogenic mechanisms of $2 \mathrm{HG}$ are uncertain, therapeutic inhibition of its production by mutant IDH1 presents an exciting possibility for slowing or halting the progression of malignant gliomas.

Sophie Atkinson

ORIGINAL RESEARCH PAPER Dang, L. et al. Cancer-associated IDH1 mutations produce 2-hydroxyglutarate. Nature 27 Nov 2009 (doi:10.1038/nature08617) 